\title{
Sequenced bioleaching and bioaccumulation of phosphorus from sludge combustion - A new way of resource reclaiming
}

\author{
J. Zimmermann ${ }^{1, a}$ and W. Dott ${ }^{2, b}$ \\ ${ }^{1,2}$ Institute of Hygiene and Environmental Health, RWTH Aachen University, Pauwelsstrasse 30, \\ D-52074 Aachen, Germany, Tel: +49 2418088883 \\ a jennifer.zimmermann@rwth-aachen.de, ${ }^{b}$ wolgang.dott@post.rwth-aachen.de
}

Keywords: bioleaching, heavy metals, phosphorus, bioaccumulation, Bio-P

\begin{abstract}
The recovery of phosphorus from sewage sludge incineration ash as well as the separation of heavy metals from ash was investigated by using the biotechnological process of bioleaching and bioaccumulation of released phosphorus by newly developed population of bioleaching bacteria, Acidithiobacillus sp. strains, and polyphosphate (poly-P) accumulating bacteria, the AEDSpopulation (Acidithiobacillus sp. enriched digested sludge). The biologically performed solubilization of phosphorus from sewage sludge incineration ash is accompanied by the release of toxic metals. Therefore a combined process to separate phosphorus from heavy metals by achieving a plant available phosphorus-enriched product and a metal depleted ash was designed. Leaching experiments were conducted in laboratory scaled leaching reactor containing a bacterial stock culture of Acidithiobacillus sp.. Next step was the enhancement of P-recovery in combining bioleaching with simultaneous bio-P-accumulation by AEDS-population. The uptake of phosphorus in biomass reaches up to $66 \%$ of the mobilized phosphorus by bioleaching. The combined biologically performed technology of phosphorus leaching and separation from toxic metals by simultaneous bioaccumulation developed in this study is a promising process for economical and ecological recovery of phosphorus from waste solids.
\end{abstract}

\section{Introduction}

The increasing global demand of plant available phosphorus leads to a scarcity of natural phosphorus (P) resources. Investigating new P-resources, sewage sludge can be regarded as promising P-sink. If sewage sludge is not suitable as fertilizer, it must be incinerated. The resulting ash is characterized as a sink for phosphorus and a concentrate of heavy metals. Therefore, technologies that allow the recovery of phosphorus from sewage sludge ashes have gained more and more interest. Leaching with inorganic acids allows recovering phosphorus but a costly separation from heavy metals is necessary. The combined process of bioleaching from phosphorus and metals by Acidithiobacillus sp. strains and the simultaneous separation of released phosphorus by Paccumulating bacteria by newly developed bacteria population named AEDS-population, an environmentally compatible procedure for waste water treatment and nutrient recovery is possible. The principal microorganism involved in bioleaching process is A. ferrooxidans, a chemolithotrophic organism. This organism mainly receives its energy from oxidation of ferrous iron to ferric iron and metal sulphides to sulphates. Another bacteria involved is A. thiooxidans, which can use elemental sulphur as substrate for growth producing sulphuric acid. This leads to a reduction in $\mathrm{pH}$-value and an increase of solved metals. Bioleaching is a promising treatment in resolving metals from sewage sludge [1,2] and fly ash [3,4]. In this project sewage sludge incineration ash was used as substrate for bioleaching. By leaching the ash, the amount of phosphorus increases as well as the dissolution of metals, and can be recovered by biological Paccumulation. This process is based on poly-P-accumulating organisms, named PAOs [5]. PAOs were characterized as mixed consortium of different sludge indigenous bacteria in enhanced biological phosphorus removal (EBPR) [6]. In order to recover $\mathrm{P}$ and separate heavy metals from sewage sludge incineration ash, a mixture of $A$. ferrooxidans and $A$. thiooxidans strains was used as 
leaching media in comparison to common chemical treatment. To get the bacteria population of Acidithiobacillus sp. strains and Poly-P bacteria, anaerobic stabilized digested sludge was used as substrate. The occurrence of PAO's is also given in digested sludge [7], but was never investigated under low $\mathrm{pH}$ conditions. Hence, the AEDS-population is the first attempt to combine a bioleaching process with a simultaneous P-recovery from sewage sludge incineration ash catalysed by microorganisms (MOs).

\section{Materials and Methods}

Experiments were conducted in a lab-scale bioleaching reactor using the following conditions: constant flow $(25 \mathrm{ml} / \mathrm{min})$ of media, medium volume of $500 \mathrm{ml}$, aeration $(20 \mathrm{l} / \mathrm{min}$ ambient air) and $2 \mathrm{~g}$ of sewage sludge incineration ash of sludge combustion in wastewater treatment plant Bonn, Germany. Sewage sludge incineration ash was leached with Acidithiobacillus sp. species, AEDSpopulation and sulphuric acid (2M) for 11 days.

\section{Acidithiobacillus sp. strains and AEDS- population}

A. ferrooxidans and A. thiooxidans (DSM 583, DSM 14887) were cultivated in media for lithotrophic organisms, $\mathrm{pH} 4.4$ - 4.7, with additional elemental sulphur. For AEDS-population enrichment, digested sewage sludge from waste water treatment plant Aachen, Germany, was incubated with added sulphur for 15 days by $22{ }^{\circ} \mathrm{C}$. When reaching $\mathrm{pH} 2.3-2.0$, sludge was centrifuged and supernatant was used as leaching medium in bioleaching reactor.

\section{Sampling and analysis}

PH was measured once a day with appropriate electrodes. Sampling of leaching medium was daily for phosphorus and metal content. Metal analysis was performed with ICP-MS (Perkin Elmer). Ion chromatography (ICS 3000, Dionex) was used to determine phosphorus as orthophosphate according to DIN 38405 method.

\section{Results and Discussion}

\section{Bioleaching of phosphorus and metals}

The behaviour of phosphorus release from sewage sludge incineration ash by bioleaching process was carried out in the initial series of experiments. Therefore, leaching experiments with (1) mixed culture of $A$. ferrooxidans and thiooxidans, (2) AEDS- population and (3) 2M sulphuric acid were performed and P-release was compared (Fig. 1). From the ash, containing $80 \mathrm{mg} / \mathrm{g} \mathrm{P}$, maximal releasing rates for $\mathrm{P}$ with bioleaching with Acidithiobacillus sp. species were $74-75 \mathrm{mg} / \mathrm{g}$ (93\%) P, $53 \mathrm{mg} \mathrm{P} / \mathrm{g}(66 \%)$ with AEDS-population and $41 \mathrm{mg} \mathrm{P} / \mathrm{g}(51 \%)$ with chemical leaching. The recovered $\mathrm{P}$ was available as orthophosphate. Metal extraction experiments were performed for Acidithiobacillus sp.-strains, AEDS-population and 2M sulphuric acid, due to the promising results in P-leaching. The aim in these bioleaching experiments was to extract metals from ash into solution as efficient as possible to get a metal depleted ash. Alike, a high metal leaching efficiency even with AEDS-population had to be verified. The results of metal leaching experimernts indicated that the metal extraction yields in mixed culture of $A$. ferrooxidans and thiooxidans achieved nearly the same amounts like AEDS-population (Fig.2). Chemical leaching showed for all examined metals higher amounts. The maximum metal extraction yields with mixed culture for $\mathrm{Fe}, \mathrm{Al}, \mathrm{Cu}$, $\mathrm{Zn}, \mathrm{Cr}$ and Co were 16, 61, 41, 20, 13 and $34 \%$, respectively. With AEDS-population metal recovery for $\mathrm{Fe}, \mathrm{Al}, \mathrm{Cu}, \mathrm{Zn}, \mathrm{Cr}$ and $\mathrm{Co}$ was 13, 55, 31, 45, 13 and 34 \%, for chemical leaching 32, $67,75,83,36$ and $35 \%$, respectively. Hence, the process of resolving phosphorus from incineration ash as well as the extraction of metals was given by using the biological process of bioleaching. Even if metal amounts of chemical leaching achieve higher yields, a costly separation of $\mathrm{P}$ from metal solution is necessary. 


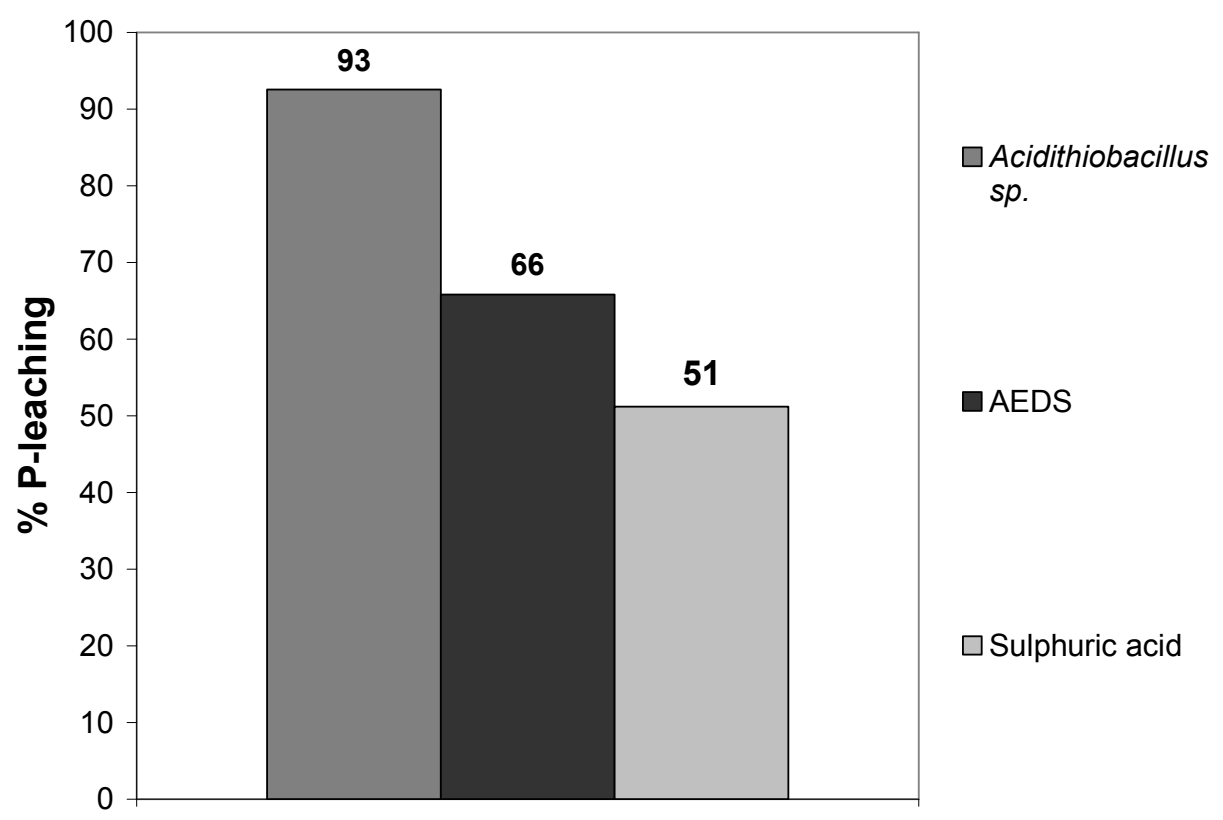

Fig. 1. Rate of phosphate-leaching of sewage sludge ash using AEDS-population, medium with Acidithiobacillus sp. and sulphuric acid 2M

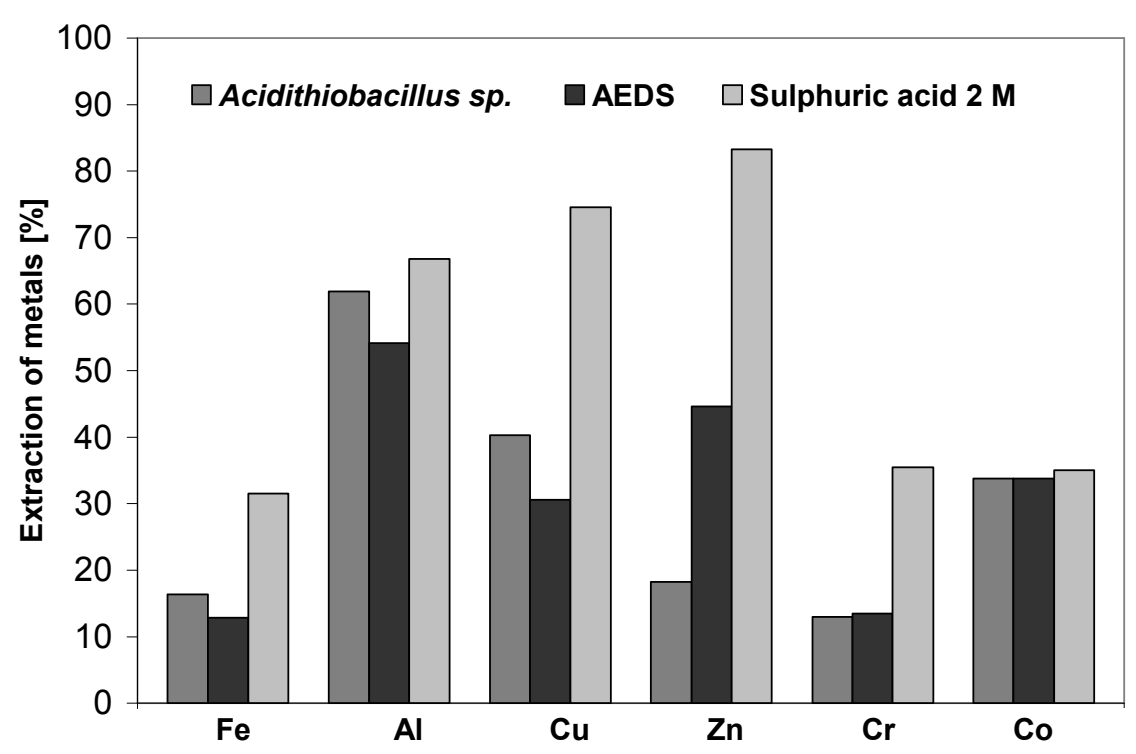

Fig. 2. Metals extraction yields in bioleaching using Acidithiobacillus sp., AEDS-population and sulphuric acid 2M.

Bioaccumulation of phosphorus and separation of heavy metals

To get a plant available phosphorus-product, released $\mathrm{P}$ must be separated from extracted metals. Therefore, the newly developed population of bacteria was applied. The experiments for observing the phosphate disposition were conducted with mixed Acidithiobacillus-culture and AEDSpopulation. After 3 days the AEDS-population started to accumulate the released phosphorus as polyphosphate and biomass increased. With mixed culture of Acidithiobacillus sp. and sulphuric acid, no significant accumulation of phosphorus was apparent. AEDS-population was able to accumulate nearly $66 \%$ of released phosphorus in biomass. The accumulated phosphorus can hereby be departed from dissolved toxic metals as plant available phosphate product (Fig.3). The fact of an existing population of leaching bacteria and PAOs in low $\mathrm{pH}$-ranges was the first attempt to recover phosphate in a single biologically performed process. 


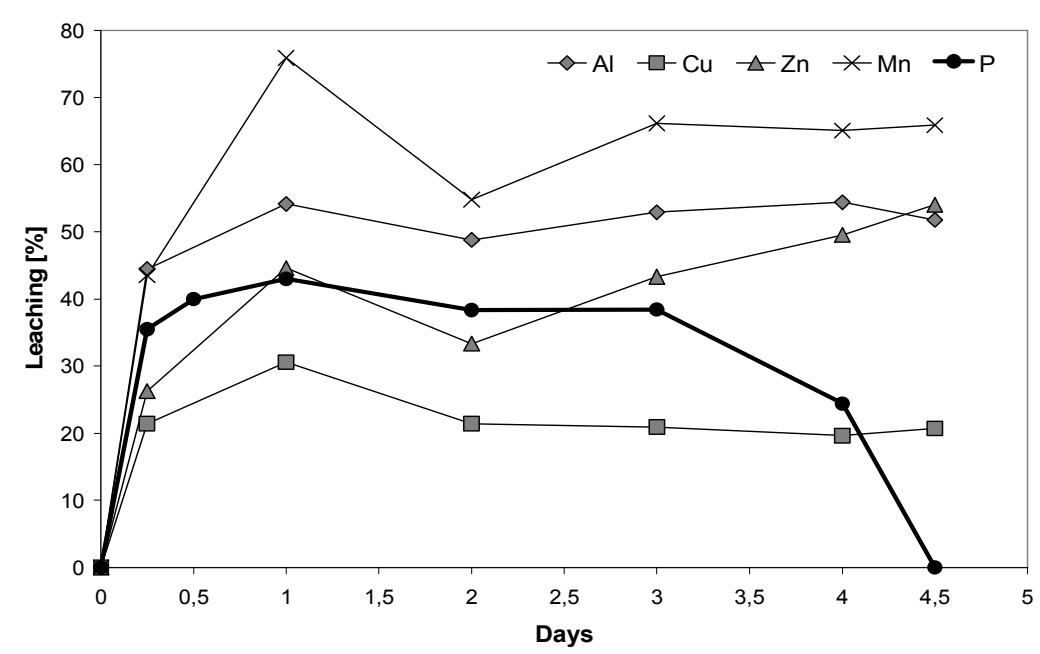

Fig. 3. Release of metals and phosphorus by bioleaching and bio-P-accumulating process.

\section{Conclusions}

This research investigated the effect of bioleaching and bioaccumulation on the recovery of phosphorus. Aside the well known chemical leaching and precipitation of phosphorus as plant available fertilizer, a simple, economical and ecological alternative was developed. The bioleaching process to release phosphorus and the contemporaneous biological phosphorus accumulation is a new way to recycle phosphorus from waste waster treatment. As leaching material sewage sludge incineration ash from sludge combustion was used. Incineration ash is a sink for phosphorus, but also for heavy metals. The treatment with Acidithiobacillus sp. solves phosphorus in higher yields than conventional chemical leaching. Equally, the yields in metal removal are adequate to perform an ecologically unobjectable ash. For separation of released phosphorus from metals, the newly developed AEDS-population is able to leach metals and phosphorus and simultaneously accumulate it as polyphosphate. Even in low pH-ranges, the association of Acidithiobacillus sp. and PAOs is possible and efficient. Hence, biologically resolved phosphorus is separated from toxic metal solution and phosphate enriched biomass can be used as plant available product.

\section{Acknowledgments}

Bioleaching studies are part of the project 'Recycling of plant nutrients - in particular phosphorus from sewage sludge ash' (PASCH) supported by BMBF. For supplying AEDS-population and funding bioaccumulation experiments AIR UMWELT GmbH (Aachen, Germany) is gratefully acknowledged.

\section{References}

[1] D. Couillard and G. Mercier: Environmental Pollution Vol. 66 (1990), p. 237

[2] D. Couillard and M. Chartier: Journal of Biotechnology Vol. 20 (1991), p. 163

[3] C. Brombacher, R. Bachofen and H. Brandl: Applied and Environmental Microbiology Vol. 64 (1998), p.1237

[4] J. Yang, Q. Wang and T. Wu: Environmental Engineering Science Vol. 25 (2008), p.783

[5] Q. Wu, P.L. Bishop and T.C. Keener: Water Environment Research Vol. 78 (2006), p. 196

[6] J. Hollender, U. Dreyer, L. Kronberger, P. Kämpfer and W. Dott: Applied Microbiology and Biotechnology Vol. 58 (2002), p.106

[7] C.M. Lopez-Vasquez, C.M. Hooijmans, D. Brdjanovic, H.J. Gijzen and M.C.M. van Loosdrecht: Water Environment Research Vol. 79 (2007), p. 2487 